Original article

\title{
Prevalence and impact of generalized anxiety disorder and major depression in primary care in Belgium and Luxemburg: the GADIS study
}

\author{
Marc Ansseau $^{\mathrm{a}, *}$, Benjamin Fischler ${ }^{\mathrm{b}}$, Michel Dierick ${ }^{\mathrm{c}}$, Annick Mignon ${ }^{\mathrm{d}}$, Sophie Leyman ${ }^{\mathrm{d}}$ \\ ${ }^{a}$ Department of Psychiatry and Medical Psychology, CHU du Sart Tilman (B35), University of Liège, 4000 Liège, Belgium \\ ${ }^{\mathrm{b}}$ University Hospital St. Pierre, Brussels, Belgium \\ ${ }^{\mathrm{c}}$ Psychiatric Hospital St. Camillus, Ghent, Belgium \\ ${ }^{\mathrm{d}}$ Wyeth Pharmaceuticals, Louvain-la-Neuve, Belgium
}

Received 15 December 2003; received in revised form 10 September 2004; accepted 14 September 2004

Available online 26 January 2005

\begin{abstract}
Purpose. - GADIS aims at determining the prevalence of generalized anxiety disorder (GAD) and major depression (MD) in primary care and their impact on the patient's functioning in Belgium and Luxemburg.

Method. - A large scale screening program was conducted at the consultation of general practitioners to detect patients with GAD and MD according to DSM-IV criteria. We collected additional data regarding the use of hypnotic, tranquilizer, antidepressant and analgesic medications. Impact on the patient was assessed with the Sheehan disability scale.

Results. - Three hundred GP's in Belgium and Luxemburg were asked to screen 50 consecutive patients. Of the 13,677 analyzed patients, $8.3 \%$ were diagnosed to have GAD and $6.3 \% \mathrm{MD}$. Comorbidity was observed in $4.2 \%$ of patients. The prevalence was much higher in the French-speaking part of Belgium. GAD and MD were associated with impairment in social, familial and professional functioning. Only a minority of patients with GAD and/or MD was treated with an antidepressant and almost half of subjects with GAD and/or MD were treated with a tranquilizer.

Conclusion. - Prevalence rates of GAD and MD in primary care in Belgium are comparable to other countries. GAD and MD are disabling conditions. Antidepressants are still used only in a minority of subjects with GAD and/or MD in primary care in Belgium and Luxemburg. The prevalence of GAD and MD appears to be much higher in French-speaking parts of Belgium.
\end{abstract}

(C) 2005 Elsevier SAS. All rights reserved.

Keywords: Depression; Anxiety; Prevalence; Primary care; Impairment; Generalized anxiety disorder; Major depression

\section{Introduction}

A number of epidemiological studies have shown that depression and anxiety disorders are highly prevalent in the general population and in primary care [10,22]. These diagnoses are, however, frequently missed due to patient related reasons such as the stigmatization of mental illness and physician related reasons such as insufficient awareness of the diagnoses, and cause these illnesses to remain untreated. Generalized anxiety disorder (GAD) is characterized by chronic worry, anxiety and tension and frequently occurs concomitantly with other disorders, mainly depression [10]. GAD and major depression (MD) are debilitating illnesses, even more

\footnotetext{
* Corresponding author. Tel.: +32 4366 7960; fax: +324366 7283.

E-mail address: marc.ansseau@chu.ulg.ac.be (M. Ansseau).
}

than other chronic illnesses such as diabetes or arthritis [20] with an important impact on the personal life of the patients and their family.

With the availability of new treatment options, there is a growing interest to detect and treat these illnesses at the primary care level.

GADIS was aimed at detecting GAD and depression in the primary care setting in Belgium and Luxemburg and also at measuring the impact of these conditions on the patient's functioning. In addition, the study aimed at describing the pharmacological treatment received by the patients.

\section{Subjects and methods}

Three hundred general practitioners were asked to participate in a large scale screening program. In order to have rep- 
resentative samples of patients consulting primary care in Belgium and Luxemburg, the investigators had to be primary care physicians, geographically distributed over Belgium and Luxemburg, with medium to large size practices. Every medical representative of the Wyeth laboratory selected five GP's in his/her sector complying with these criteria.

The validated French and Dutch translations of GAD and major depressive episode (MD) sections from the Mini Neuropsychiatric Interview (MINI) were used with permission of the authors to make the diagnoses of GAD and MD according to DSM-IV criteria [16]. Physicians were not trained in the use of the MINI.

In order to avoid bias in the selection of the patients, the participating GP determined the dates of the consultations at their office at which all consecutive patients were going to be screened. Between November 2001 and March 2002, each physician screened 50 consecutive patients of at least 18 years who presented at their consultation at the previously fixed time periods.

The patient was interviewed by the GP with the GAD and MD sections of the MINI [16]. The Case Report Form, filled by the GP, captured the reason for consultation, the current hypnotic, tranquilizer, antidepressant or analgesic medications.

In order to make the study feasible for the majority of GP's, 250 GP's were asked to collect the information on treatment and disability only in patients that screened positive for GAD or MD, whereas 50 GP's were asked to collect all information in all their screened subjects. The latter group of GP's thus generated the information for the control group of subjects without GAD and MD, on use of medication and patient's functioning. Most characteristics were found to be similar between both groups of patients (age, region, reason for consultation). Both groups differed significantly $(P$ $=0.043$ ) for gender with a slightly higher proportion of women in the latter group (57.2\%) compared with the former group $(55.0 \%)$.

Impact on the patient's functioning was assessed using the Sheehan disability self-assessment scale [15].

Finally, the protocol was approved by the Ethics Committee of the Catholic University of Leuven and patients were required to provide written informed consent prior to the screening.

\section{Statistics}

In tables, results are expressed as mean \pm standard deviations (S.D.) for quantitative variables and as counts and proportions (prevalence) for categorical findings. Prevalence rates were associated with their $95 \%$ confidence intervals whenever needed. Mean values were compared by classical oneway analysis of variance (ANOVA) and proportions by the $\chi^{2}$-square test for contingency tables. Non parametric Kruskal-Wallis and Wilcoxon tests were also used for comparing samples from different groups. Correlation coeffi- cients (classical or non parametric Spearman) were calculated to measure the association between continuous variables. General linear models were used to measure the effect of several "independent" factors on a "dependent" variable. Logistic regression analysis was used to assess the relationship between a binary outcome variable (e.g. GAD or MD) and a set of independent variables. The approach was also utilized to derive relative risk (RR) or odds ratio (OR) quantities. All results were considered to be statistically significant at the $5 \%$ level $(P<0.05)$ (two-tailed). Statistical analyses were always carried out on the maximum number of data available (pair-wise deletion of missing data which explains slight differences of $\mathrm{N}$ in some tables) using the SAS (version 8.2 for Windows) and S-Plus (version 6.0) software packages.

\section{Results}

\subsection{Study population}

Fifteen thousand $(15,000)$ patients were expected to be screened. However, not all GP's included 50 patients and a number of files were excluded because of the large number of missing data. Ultimately, a sample of 13,677 patients consulting their general practitioner in Belgium and Luxemburg were included in the analyses.

\subsection{Characteristics of the patient population}

Detailed characteristics of patients are shown in Table 1.

A significant age difference was observed between men and women $(50.4 \pm 17.0$ vs. $48.8 \pm 17.4$ years, respectively; $P<0.0001)$. Regions were homogeneous with respect to gender (data not shown) but not to age $(P<0.0001)$. Patients were older in Brussels $(52.3 \pm 17.8$ years $)$ than in Wallonia (49.6 \pm 17.1 years), Flanders $(49.1 \pm 17.2$ years $)$ and Luxemburg $(45.8 \pm 16.7$ years $)$.

Reasons for consulting the GP were not the same in the regions $(P<0.0001)$. The proportion consulting for anxiety or depression was significantly lower in Flanders (3.4\%) and

Table 1

Characteristics of the patient population $(n=13677)$

\begin{tabular}{ll}
\hline Variable & $\begin{array}{l}\text { Mean } \pm \text { S.D. } \\
\text { (or number and proportions) }\end{array}$ \\
\hline $\begin{array}{l}\text { Age (years) } \\
\text { Gender }\end{array}$ & $49.8 \pm 17.3$ \\
Female & $7510(55.4 \%)$ \\
Male & $6054(44.6 \%)$ \\
Region & \\
Flanders & $6937(50.9 \%)$ \\
Wallonia & $4638(34.1 \%)$ \\
Brussels & $1501(11.0 \%)$ \\
Luxemburg & $542(4.0 \%)$ \\
Reason for consultation & \\
Chronic disease & $5636(44.4 \%)$ \\
New illness & $6467(50.9 \%)$ \\
Anxiety or depression & $600(4.7 \%)$ \\
\hline
\end{tabular}


Luxemburg (2.2\%) than in Wallonia (6.6\%) and Brussels (5.7\%). Reasons for consulting were also significantly related to gender $(P<0.0001)$ and age $(P<0.0001)$ with a significantly higher proportion of women consulting for anxiety and depression (67\%) than for chronic disease $(54 \%)$ or new illness $(56 \%)$. As expected, patients consulting for a chronic disease were found to be older $(59.1 \pm 15.0$ years $)$ than those consulting for anxiety or depression $(44.3 \pm 12.8$ years $)$, themselves being older than those presenting with a new illness $(41.7 \pm 15.2$ years $)$.

\subsection{Overall prevalence of $G A D$ and/or $M D$}

Table 2 gives the prevalence of GAD and MD in the entire patient population.

The prevalence of GAD alone $(4.1 \%)$ was about twice as high as MD alone $(2.1 \%)$. The prevalence of the combination of the two disorders reached $4.2 \%$. Overall, $10.4 \%$ of the patients were diagnosed as GAD and/or MD.

\subsection{Prevalence according to gender}

As seen in Table 3, there was a significant effect of gender on the prevalence of GAD alone $(P<0.0001)$ and to a lesser extent on MD alone $(P=0.029)$. In each case, prevalence in women was significantly higher than in men. The RR varied between 1.3 and 2.0.

\subsection{Prevalence according to age}

Fig. 1 clearly demonstrates a quadratic evolution $(P<0.0001)$ of the prevalence of GAD, which increased until

Table 2

Prevalence of MD and GAD $(n=13480)$

\begin{tabular}{llcl}
\hline Disorder & Number & Prevalence & $95 \%$ CI \\
\hline GAD alone & 555 & $4.1 \%$ & $3.78-4.45$ \\
MD alone & 288 & $2.1 \%$ & $1.89-2.38$ \\
GAD + MD & 565 & $4.2 \%$ & $3.85-4.53$ \\
All GAD & 1120 & $8.3 \%$ & $7.84-8.77$ \\
All MD & 853 & $6.3 \%$ & $5.92-6.74$ \\
Total & 1408 & $10.4 \%$ & $9.93-11.0$ \\
\hline
\end{tabular}

Table 3

Prevalence rates of GAD and MD according to gender

\begin{tabular}{lllcc}
\hline Disorder & $\begin{array}{l}\text { Women, } \\
n=7404\end{array}$ & $\begin{array}{l}\text { Men, } \\
n=5965\end{array}$ & $P$-value & RR \\
\hline GAD alone & $385(5.20 \%)$ & $167(2.80 \%)$ & $<0.0001$ & 1.86 \\
MD alone & $176(2.38 \%)$ & $109(1.83 \%)$ & 0.029 & 1.30 \\
GAD + MD & $400(5.40 \%)$ & $159(2.67 \%)$ & $<0.0001$ & 2.03 \\
Total & $961(13.0 \%)$ & $435(7.29 \%)$ & $<0.0001$ & 1.78 \\
\hline
\end{tabular}

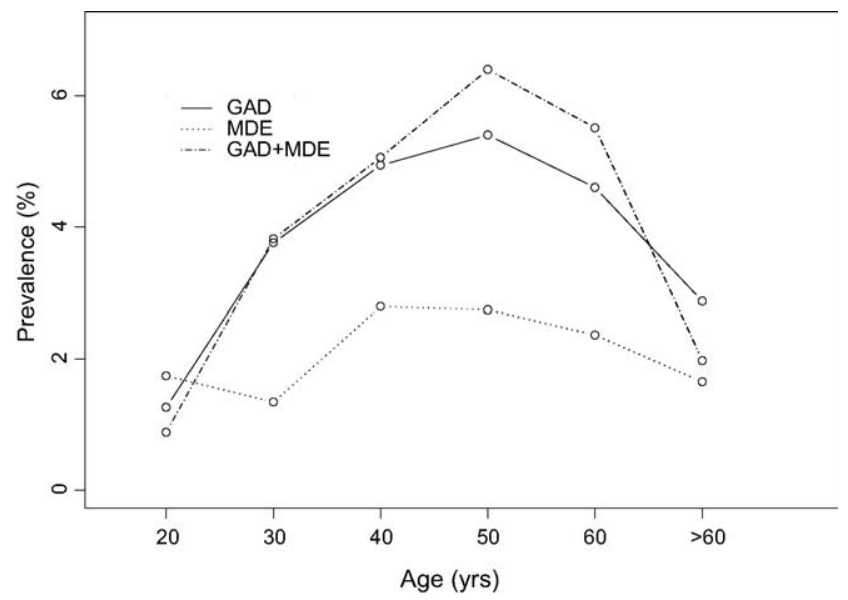

Fig. 1. Distribution of GAD and MD according to age.

the age of 50 years and thereafter dropped to lower values. The prevalence of MD follows the same trend pattern but less strikingly $(P<0.0017)$.

\subsection{Prevalence according to regions}

Highly significant differences in GAD/MD prevalence rates were observed between regions $(P<0.0001)$ (Table 4$)$.

Prevalence rates were significantly higher in Wallonia, Brussels and even in Luxemburg than in Flanders, confirming the differences observed above in the reasons for consulting. Overall prevalence rates reached $13.1 \%$ in Wallonia, $13.6 \%$ in Brussels and $11 \%$ in Luxemburg, but only $7.9 \%$ in Flanders.

\subsection{Prevalence in patients consulting for anxiety/depression}

The prevalences of GAD and MDD in the 600 patients (4.7\% of the total sample) who reported anxiety/depression as reason for consulting their GP is displayed in Table 5. A total of $84.6 \%$ of those patients were positive for at least one of the two disorders. No difference existed regarding prevalence rates according to gender in this subsample.

\subsection{Existing treatment}

Among patients with a positive diagnosis (GAD alone, MD alone or GAD + MD), $48 \%$ were being treated with tranquilizers at the time of the visit to the general practitioner. The proportions were $32 \%$ for antidepressants, $27 \%$ for hypnotics, and $24 \%$ for analgesics, respectively. Differences were

Table 4

Prevalence rates of GAD and MD according to regions

\begin{tabular}{|c|c|c|c|c|c|}
\hline Disorder & Flanders, $n=6842$ & Wallonia, $n=4544$ & Brussels, $n=1498$ & Luxemburg, $n=539$ & $P$-value \\
\hline GAD alone & $216(3.2 \%)$ & $229(5.0 \%)$ & $71(4.7 \%)$ & $29(5.4 \%)$ & $<0.0001$ \\
\hline MD alone & $116(1.7 \%)$ & $107(2.3 \%)$ & $55(3.7 \%)$ & $10(1.9 \%)$ & $<0.0001$ \\
\hline $\mathrm{GAD}+\mathrm{MD}$ & $206(3.0 \%)$ & $258(5.7 \%)$ & $78(5.2 \%)$ & $20(3.7 \%)$ & $<0.0001$ \\
\hline Total & $538(7.9 \%)$ & $594(13.1 \%)$ & $204(13.6 \%)$ & $59(11.0 \%)$ & $<0.0001$ \\
\hline
\end{tabular}


Table 5

Prevalence of GAD and MD in patients consulting for anxiety/depression ( $n$ $=600)$

\begin{tabular}{llll}
\hline Disorder & Number & Prevalence $(\%)$ & $95 \%$ CI \\
\hline GAD alone & 135 & 22.7 & $17.0-28.4$ \\
MD alone & 89 & 15.0 & $10.1-19.9$ \\
GAD + MD & 279 & 46.9 & $40.2-53.4$ \\
All GAD & 417 & 69.6 & $58.2-81$ \\
All MD & 369 & 61.9 & $54.6-69.2$ \\
Total & 503 & 84.6 & $77.0-92.2$ \\
\hline
\end{tabular}

found between diagnostic categories for hypnotics, tranquilizers and antidepressants but not for analgesics (Table 6). For all types of medication, drug use was higher in patients suffering from comorbid GAD/MD.

The average number of drugs taken per day varied between 2.3 and 2.7 (average $2.5 \pm 2.1$ ) and a significant difference was observed between GAD alone and GAD + MD $(P=0.0065)$, with a higher number of drugs for the latter.

When comparing "positive" patients (for GAD/MD) with patients from the control group (see Section 2), we found significantly higher proportions of drug use in the former group, even after adjusting for cofactors like gender, age and region $(P<0.0001)$ (Table 7$)$. Moreover, the number of types of drugs taken was also higher in the former group $(P<0.0001)$.

\subsection{Impact on patient}

On the Sheehan disability scale, the patient rates the degree of impairment from 0 to 10 in three domains of his/her life: work, social life and family life. Higher scores indicate higher levels of impairment. Results on the Sheehan disability scale are given in Table 8, not only for each diagnostic category but also for patients with or without GAD and/or MD.

Patients with GAD and/or MD had very significantly higher item scores than control patients $(P<0.0001)$. Also, patients combining the two disorders had higher scores than those affected by a single disorder.

Correlations between all items of the Sheehan disability scale were highly significant (data not shown).

The $P$-values between the three diagnostic groups were all $<0.0001$ and the $P$-values between positive and negative patients were all $<0.0001$.

In order to exclude a possible effect of gender and age upon the association of diagnosis and disability, a multiple regression analysis was conducted with disability as dependent variable and diagnosis, gender and age as independent variables. No effect of age was observed. A statistically significant independent effect was found for diagnosis, gender for the three Sheehan subscales (respectively work: diagnosis, $P<0.0001$; gender, $P=0.0014$; social: diagnosis, $P<0.0001$; gender, $P=0.0016$; familial: diagnosis, $P<0.0001$; gender, $P=0.0046)$. The interaction between

Table 6

Current medications in patients with positive diagnosis of MD and/or GAD

\begin{tabular}{|c|c|c|c|c|c|}
\hline Drug & $\begin{array}{l}\text { GAD alone, } \\
n=555\end{array}$ & $\begin{array}{l}\text { MD alone, } \\
n=288\end{array}$ & $\begin{array}{l}\text { GAD + MD, } \\
n=565\end{array}$ & $P$-value & $\begin{array}{l}\text { Total, } \\
n=1408\end{array}$ \\
\hline Hypnotics & $125(23 \%)$ & $75(27 \%)$ & $163(31 \%)$ & 0.025 & $363(27 \%)$ \\
\hline Tranquilizers & $236(44 \%)$ & $115(40 \%)$ & $306(55 \%)$ & $<0.0001$ & $657(48 \%)$ \\
\hline Antidepressants & $130(24 \%)$ & $83(29 \%)$ & $132(42 \%)$ & $<0.0001$ & $445(32 \%)$ \\
\hline Analgesics & $116(23 \%)$ & $58(22 \%)$ & $138(27 \%)$ & 0.13 & $312(24 \%)$ \\
\hline Number drugs per day & $2.3 \pm 2.0^{\mathrm{a}}$ & $2.6 \pm 2.2$ & $2.7 \pm 2.1^{\mathrm{a}}$ & 0.0065 & $2.5 \pm 2.1$ \\
\hline
\end{tabular}

${ }^{\text {a }}$ Scheffé test.

Table 7

Current medications in patients with positive and negative GAD and/or MD diagnoses

\begin{tabular}{lllll}
\hline Drug & $\begin{array}{l}\text { Positive patients, } \\
n=1408\end{array}$ & $\begin{array}{l}\text { Negative patients, } \\
n=2354\end{array}$ & OR & 95\% CI \\
\hline Hypnotics & $363(27 \%)$ & $204(11 \%)$ & 3.12 & $2.58-3.77$ \\
Tranquilizers & $657(48 \%)$ & $273(14 \%)$ & 5.51 & $4.67-6.50$ \\
Antidepressants & $445(32 \%)$ & $120(6.2 \%)$ & 7.15 & $5.76-8.88$ \\
Analgesics & $312(24 \%)$ & $321(17 \%)$ & 1.59 & $1.33-1.89$ \\
Number drugs per day & $2.5 \pm 2.1 *$ & $1.9 \pm 2.0$ & - & - \\
\hline
\end{tabular}

$* P<0.0001$.

Table 8

Sheehan disability scale scores in positive and negative patients for MD and/or GAD

\begin{tabular}{|c|c|c|c|c|c|}
\hline Scale & $\begin{array}{l}\text { GAD alone, } \\
n=555\end{array}$ & $\begin{array}{l}\text { MD alone, } \\
n=288\end{array}$ & $\begin{array}{l}\text { GAD + MD, } \\
n=565\end{array}$ & $\begin{array}{l}\text { Positive patients, } \\
n=1408\end{array}$ & $\begin{array}{l}\text { Negative patients, } \\
n=2354\end{array}$ \\
\hline \multicolumn{6}{|c|}{ Disability } \\
\hline Work & $5.8 \pm 2.3$ & $6.3 \pm 2.2$ & $7.1 \pm 1.9$ & $6.4 \pm 2.2$ & $1.7 \pm 2.1$ \\
\hline Social & $5.8 \pm 2.3$ & $6.3 \pm 2.3$ & $7.3 \pm 1.9$ & $6.5 \pm 2.2$ & $1.4 \pm 2.0$ \\
\hline Family & $5.8 \pm 2.4$ & $6.2 \pm 2.3$ & $7.3 \pm 2.0$ & $6.5 \pm 2.3$ & $1.4 \pm 2.0$ \\
\hline
\end{tabular}


gender and diagnosis was found to be statistically significant for the subscale work functioning $(P=0.04)$, marginally significant for social functioning $(P=0.06)$ and not significant for familial functioning. This means that independently of a gender effect on disability, diagnosis of MD and/or GAD has a separate contribution to disability. Furthermore, women with GAD and/or MD were found to be more professionally disabled than men with GAD and/or MD.

The levels of impairment were clearly different in patients with or without GAD and/or MD. The distribution of patients according to the score on the Sheehan disability scale showed that most patients who were positive for GAD and/or MD had scores of 5 or higher. Patients who did not receive a diagnosis of GAD or MD rarely had scores higher than 4 .

Of the positive patients $83 \%$ have a score of 5 or more on the work item compared to $12 \%$ of the control patients. For the social function, $83 \%$ of positive patients and $10 \%$ of control patients have a score of at least 5 and regarding family function $80 \%$ of positive patients have a score of at least 5 compared to $10 \%$ of control patients.

\section{Discussion}

\subsection{Prevalence}

The prevalence of GAD obtained in the current study (8.3\%) compares well with the literature where the prevalence of GAD in primary care ranges from $6.4 \%$ to $14.9 \%$ but approximates $8 \%$ in several studies [8,14,19,21]. It is somewhat higher than in the general population, confirming that patients suffering from GAD and MD more frequently consult the primary care physician. A recent Belgian study using a different screening instrument (Prime-MD) in 2316 patients showed a higher prevalence for generalized anxiety $(10.3 \%)$ and a much higher prevalence of MD (13.9\% as compared with $6.3 \%$ in the present study) [1]. These differences in a similar population can be due to the inclusion of patients seen during home visits (29.6\% of the sample) in this previous study. These patients who are unable or unwilling to attend the GP office could be characterized by more psychiatric disturbances.

The point prevalence obtained in GADIS only detected the patients who have currently all the diagnostic criteria for GAD and MD. They do not include the patients with a prior diagnosis who no longer fulfill all the criteria because they receive adequate treatment (partial or complete remission) neither subjects with a subsyndromal condition.

In the screened population, $4.7 \%$ of the patients came to see their GP because of anxiety or depression whereas in $10.4 \%$ a new diagnosis was made. This possibly means that many patients with GAD and/or MD are not recognized by their GP's. Another possibility (which does not exclude the former) is that GAD and or MD is prevalent in patients with organic conditions and that therefore these patients are not classified by their GP as consulting for anxiety and/or depres- sion. A third possibility is the fact that many anxious and depressive patients present with somatoform complaints [5]. In this subgroup, a diagnosis of GAD or MD was made in $84.6 \%$ of patients, with slightly higher rate of GAD as compared to MD (69.6\% vs. $61.9 \%)$ and both diagnoses present in about half of the subjects. Surprisingly, $15.4 \%$ of those patients did not received any diagnosis. They probably did not fulfill all criteria for a DSM-IV diagnosis despite their specific complaints.

GADIS confirms that more women than men suffer from generalized anxiety and from depression. The higher prevalence in women is particularly pronounced for GAD and GAD + depression. Also significant, the difference between men and women for depression without GAD is much less marked. This finding coincides with the conclusion of the review of Simonds and Whiffen [17]. However, a consultation bias can not be excluded in our study.

The prevalence varies according to age. For GAD the prevalence raises between the age of 20 and 50 and declines after 60. MD prevalence also increases with age but less strikingly. In previous studies in primary care the highest frequency of GAD was found between 35 and 60 years [21]. The GADIS data are in agreement with these results. The reason why the prevalence is lower in older patients can be due to inclusion of patients coming to the consultation of the general practitioner. Many older patients are visited at home by their doctor. Sometimes this is due to problems of mobility but this could also result from anxiety and depression. If GADIS would have included patients seen at their homes the prevalence may have been somewhat higher. Most data, however, indicate a decrease in prevalence after 60 years of age. The lower prevalence of GAD and MD in the elderly may be explained by the fact that older persons often live in nursing homes. They do not usually go to the physician's office but instead call upon the GP whenever needed.

GADIS shows quite impressive regional differences in prevalence. More patients in Wallonia and Brussels have a positive diagnosis for GAD and/or MD than patients in Flanders. The use of psychotropic medication is also higher in the French-speaking parts of Belgium. These results confirm previous data demonstrating extremely high prevalence of anxiety and affective disorders in two French-speaking Belgian provinces [13]. GADIS did not capture information that may explain these regional differences. Possible reasons include differences in socio-economic factors between the regions. The economic conditions between Belgian regions are very different, for example the percentage of unemployment is $16.7 \%$ in Wallonia, 20.3\% in Brussels and $7.5 \%$ in Flanders (Belgian Government, 2002). However, there are data pointing possibly to socio-cultural rather than to socioeconomic factors since French-speaking communities in Switzerland or Canada have a higher utilization of tranquilizer medications compared to other communities living in the same socio-economic environment [2]. In addition, several recent studies have shown that the use of anxiolytic and hypnotic drugs is much higher in France and Belgium than in neigh- 
boring countries $[7,9,11]$. For example, according to Pelissolo et al. [11], 25-30\% of the French general population are occasional or regular users of anxiolytic and hypnotic drugs with between 5\% and 7\% chronic users, corresponding to two to three times superior to most industrialized countries. In the survey by Ohayon and Lader [9], France had the highest proportion of anxiolytic users $(9.0 \%)$ followed by Italy (5.8\%); the rate was only $0.7 \%$ in Germany and $0.6 \%$ in the UK. It should be noted that France and Belgium are characterized by a more frequent recourse to most types of medications, reflecting probably differences in the attitude toward illness and to differences in medical education. Future screening programs should collect information on socio-economic factors such as marital status, level of education and employment in order to understand better differences in psychopathology between Belgian regions. However, it can not totally be excluded that differences in health-seeking behavior rather than psychopathology per se differ between Belgian regions. The proportion of diagnosed and not diagnosed patients who presented with anxiety/depression as reason for consultation in Dutch vs. French-speaking regions has been compared but does not show any significant differences.

\subsection{Impact}

Regarding treatment in the screened population it is not surprising to see that one in three positive patients use hypnotics, tranquilizers and/or antidepressants. The presence of both diagnoses leads to higher frequencies of psychotropic medication than in isolated disorders.

GADIS shows that approximately $10 \%$ of primary care patients without depression or GAD take hypnotics and 14\% take tranquilizers. In patients with $\mathrm{MD}$ or GAD this rate increases to $27 \%$ for hypnotics and $48 \%$ for tranquilizers. Although the present study confirms the high use of benzodiazepines [12], it also shows that most of the patients with MD do not receive the most appropriate treatment. Only $32 \%$ of the patients that have depression or GAD receive an antidepressant at the time of the consultation. It is well established that antidepressants are the most indicated pharmacological treatment for depression in primary care. More recently, antidepressants have become the appropriate pharmacological long-term treatment for chronic anxiety disorders such as GAD [3]. It should be mentioned however that non-pharmacological approaches, such as cognitivebehavioral therapies have demonstrated interest in the treatment of moderately severe forms of GAD and MD [4]. One of the several explanations of a high use of benzodiazepines in Belgium appears to be the prescription of BZD in a large number of subjects with anxiety and/or depression. Indeed, France and Belgium are characterized by extremely high benzodiazepine use. Unfortunately, we did not collect in our study precise data about the type of anxiolytic consumption (duration, occasional or chronic intake...).

The results from the patient rating Sheehan disability scale show that the presence of GAD and/or MD are associated with substantial impairment of social, professional and family functioning. This confirms that GAD and MD are disabling conditions $[6,18]$.

The majority of positive patients have both GAD and depression, confirming results from other studies. The patients with GAD and depression are clearly more severely ill, with higher scores on the Sheehan disability scale.

\subsection{Limitations}

Several limitations in the design of the study should be acknowledged. First, the selection process of GP was not randomized and could have included GP's particularly interested in psychiatric patients. Therefore, the sample of patients could eventually have been biased. It should be noted however that the size of the sample is rather large and that the demographical characteristics of the patients correspond reasonably well with those of the Belgian population. Second, the GP's were not trained in the use of the MINI. It should be noted however that only the GAD and MD section of the MINI were used and that the instrument appears rather simple. However, no inter-rater-reliability checks were obtained. Third, the MINI is potentially over-inclusive concerning GAD diagnoses because of the lack of a systematic and structured exploration of differential diagnoses (affective disorders, anxiety disorders, general medical conditions) in case of comorbidity.

\section{Conclusion}

The lessons learned from GADIS are that GAD and MD are frequent and debilitating illnesses that can easily be detected, using the right instruments. Many GP told afterwards (no formal assessment) that they have learned a lot about the diagnosis of MD and GAD with the use of this scale. Clearly, in primary care in Belgium and Luxemburg, basic axis-I diagnosis for MD and GAD remains a problem as well as the under prescription of antidepressants and the over prescription of benzodiazepines.

\section{Acknowledgments}

The authors wish to thank Professor Adelin Albert for his excellent statistical input and analysis.

\section{References}

[1] Ansseau M, Dierick M, Buntinckx F, Cnoeckaert P, De Snedt J, Van Den Haute M, et al. High prevalence of mental disorders in primary care. J Affect Disord 2004;78:49-55.

[2] Féline A, Le Goc I. Les Benzodiazépines: prescription, consommation, addiction. Sem Hop 1985;61:3172-5. 
[3] Gelenberg AJ, Lydiard RB, Rudolph RL, Aguiar L, Haskins JT, Salinas E. Efficacy of venlafaxine extended-release capsules in nondepressed outpatients with generalized anxiety disorder. JAMA 2000; 283:3082-8.

[4] Gould RA, Otto MW, Pollack MH, Yap L. Cognitive behavioral and pharmacological treatment of generalized anxiety disorder: a preliminary meta-analysis. Behav Ther 1997;28:200-10.

[5] Katon WJ, Walker EA. Medically unexplained symptoms in primary care. J Clin Psychiatry 1998;59(Suppl 20):15-21.

[6] Kessler RC, Du Pont RL, Berglund P, Wittchen HU. Impairment in pure and comorbid generalized anxiety disorder and major depression at 12 months in two national surveys. Am J Psychiatry 1999;156: 1915-23.

[7] Legrain M, Lecomte T. La consommation des psychotropes en France et dans quelques pays européens. Bull Acad Natl Med 1997;181: 1073-84.

[8] Maier W, Gaensicke M, Freyberger HJ, Linz M, Henn R, Lecrubier Y. Generalized anxiety disorder (ICD-10) in primary care from a crosscultural perspective: a valid diagnostic entity? Acta Psychiatr Scand 2000;101:29-36.

[9] Ohayon MM, Lader MH. Use of psychotropic medication in the general population of France, Germany, Italy, and the United Kingdom. J Clin Psychiatry 2002;63:817-25.

[10] Ormel J, Von Korff M, Ustun B, Pini S, Korten A, Oldehinkel T. Common mental disorders and disability across culture: results from the WHO collaborative study on psychological problems in general health care. JAMA 1994;272:1741-8.

[11] Pelissolo A, Boyer P, Lepine JP, Bisserbe JC. Epidémiologie de la consommation des anxiolytiques et des hypnotiques en France et dans le monde. Encephale 1996;22:187-96.

[12] Pestiaux JL, Marques M, Ketser F, Prévost M, Peters A. Représentation populaire et consommation de tranquilisants. Union Nationale des Mutualités Socialistes, Promotion de la Santé; 1990.
[13] Reggers J, Ansseau M. Lifetime prevalence of DSM-IV psychiatric disorders in the Liège study: preliminary results. Abstracts of the VIII Congress of the International Federation of Psychiatric Epidemiology. 1999.

[14] Sartorius N, Ustun TB, Lecrubier Y, Wittchen HU. Depression comorbid with anxiety: results from the WHO study on the psychological disorders in primary health care. Br J Psychiatry 1996;168(Suppl 30):38-43.

[15] Sheehan DV, Harnett-Sheehan K, Raj BA. The measurement of disability. Int Clin Psychopharmacol 1996;11(Suppl 3):89-95.

[16] Sheehan DV, Lecrubier Y, Sheehan KH, Amorim P, Janavs J, Weiller E. The Mini-International Neuropsychiatric Interview (MINI): the development and validation of a structured diagnostic psychiatric interview for DSM-IV and ICD-10. J Clin Psychiatry 1998;59(Suppl 20):22-33.

[17] Simonds VM, Whiffen VE. Are gender differences in depression explained by gender differences in comorbid anxiety? J Affect Disord 2003;77:197-202.

[18] Souetre E, Lozet H, Cimarosi I, Martin P, Chigon JM, Adès J. Cost of anxiety disorders: impact of comorbidity. J Psychosom Res 1994;38: 151-60.

[19] Ustün TB, Sartorius N, editors. Mental illness in general health care: an international study. Chichester: Wiley; 1995.

[20] Weiller E, Bisserbe JC, Maier W, Lecrubier Y, et al. Prevalence and recognition of anxiety syndromes in five European primary care settings: a report from the WHO study on psychological problems in general health care. Br J Psychiatry 1998;173(Suppl 34):18-23.

[21] Wittchen HU. Generalized anxiety disorder: prevalence, burden and cost to society. Depression Anxiety 2002;16:162-71.

[22] Wittchen HU, Zhao S, Kessler RC, Eaton WW. DSM-III-R generalized anxiety disorder in the national comorbidity survey. Arch Gen Psychiatry 1994;51:355-64. 